\title{
PROPOSING A SPECIFICATION STRUCTURE FOR COMPLEX PRODUCTS IN MODEL-BASED SYSTEMS ENGINEERING (MBSE)
}

\author{
Fahl, Joshua; \\ Hirschter, Tobias; \\ Wöhrle, Gabriel; \\ Albers, Albert \\ Karlsruhe Institute of Technology (KIT) / IPEK - Institute for Product Engineering
}

\begin{abstract}
This research work presents a methodological support for the specification of complex products. This is achieved by developing a specification structure in a MBSE environment. The new method draws on success factors of complex product specification, principles of MBSE and the explanatory model of PGE - Product Generation Engineering. For evaluation, the method is applied within a student development project. A high applicability and the realization of novel synergies for coping with continuously increasing product complexity is demonstrated.
\end{abstract}

Keywords: Product modelling / models, Systems Engineering (SE), Complexity, MBSE, Model of PGE - Product Generation Engineering

\section{Contact:}

Fahl, Joshua

Karlsruhe Institute of Technology

IPEK - Institute for Product Engineering

Germany

joshua.fahl@partner.kit.edu 


\section{INTRODUCTION}

In many industries, stakeholder requirements for products are increasing in terms of objectives such as functionality, quality and cost efficiency. This leads to a general increase in product complexity and presents developers with new challenges to ensure effective and efficient product development (Arslan et al., 2016). A lack of mastery of complexity can have consequences for companies such as reduced transparency and efficiency of the value chain and consequently reduce competitiveness (Schuh and Schwenk U, 2001). The specification, as the central product description, plays a critical role in the development process for the realization of the requirements (Nellore et al., 1999) and can, by using an appropriate methodology, contribute in a decisive way to making product and process complexity controllable. The development approach of Model-Based Systems Engineering (MBSE) offers the potential for better control of complexity in the development process by using a central, visualized and consistent system model for product specification (D'Ambrosio and Soremekun, 2017). Existing MBSE methods usually use standardized languages such as SysML for reasons of reproducibility. In practice, however, learning SysML represents a high entry hurdle, making the introduction and use of MBSE in companies difficult (Alt, 2019). Further potential for mastering complexity in the development process is offered by the explanatory model of PGE - Product Generation Engineering. This model describes product development as a variation of existing elements of a reference system (Albers et al., 2019) and thus supports the targeted use of existing knowledge in product specification. Based on previous findings, results and insight on product specification in literature, the present work aims to integrate and combine existing research approaches in an easy and universally applicable specification framework. Furthermore, the objective is to generate novel synergies for the management of product complexity. The following chapters are structured as follows: First, an understanding of the current state of research regarding the specification of complex products in the model of PGE as well as the validation accompanying the development and comparable MBSE methods is created. After discussing the research design, the developed specification structure is derived in the form of a MBSE approach as a novel methodological support. Based on the application of the method in a realistic development project lasting several months, the answers to the research questions are then critically discussed. Finally, a conclusion and outlook for further research concludes this contribution.

\section{STATE OF RESEARCH}

\subsection{Specification of Complex Products in the Model of PGE - Product Generation Engineering}

The Model of PGE - Product Generation Engineering according to Albers et al., 2015 describes product development on the basis of the principal assumption of a systematic use of already existing reference system elements (RSE) as a basis for the development of a new product. RSE are bundled in the reference system (Albers et al., 2019) and can be transferred into a new development project by three different variation types (Albers et al., 2020b; Albers et al., 2015): In the case of a carry-over variation $(\mathrm{CV})$, the existing element is taken over completely with only the adaptation of required interfaces. The attribute variation (AV) characterizes the use of an existing principle by adapting the characteristic values of the corresponding RSE, for example in the form of the physical shape. Finally, principle variation (PV) describes the case of a change of the underlying principle or the transfer of a RSE into another context. Product developers generally strive for a low proportion of new developments, while ensuring sufficient innovative strength, in order to reduce the effort and risks resulting from unknown interactions (Ehrlenspiel and Meerkamm, 2013). This is particularly true for complex products, which are usually characterized in system theory by the inclusion of a wide range of elements with multiple interactions (Patzak, 1982; Simon, 1994). As a consequence, product development is mainly based on incremental innovations of individual subsystems instead of fundamental changes on the level of the overall system (Deubzer and Lindemann, 2009). The product specification plays a central role in the development process. As a formal description of the product (Smith and Reinartsen, 1991), the specification serves as an artifact in the development process to define essential requirements with regard to the technical implementation (Albers et al., 2017b). At the end of the Early Phase in the model of PGE, which begins with the initiation of the project and ends with an evaluated technical solution (Albers et al., 2017b), the specification is the documentation of relevant goals on which further development work is based (Albers et al., 2013). This enables an 
evaluation of the product as well as communication/negotiation between customer and contractor (Nellore and Söderquist, 2000). As an open medium, the specification can be continuously adapted and expanded in the further course of development (Darlington and Culley, 2002). In addition to the specification as an artifact, the specification is also called an activity within the process of its creation (Nellore and Söderquist, 2000). To illustrate the character of the specification as artifact and activity as well as the link to development goals, the term can be visualized by a classification in the Extended System Triple Approach for product development (see Figure 1), which divides a product into the systems of objectives, operation system and system of objects (Albers, 2010). The specification as an activity is part of the operation system and links the system of objectives with the specification as an artifact in the system of objects.

System of Objectives

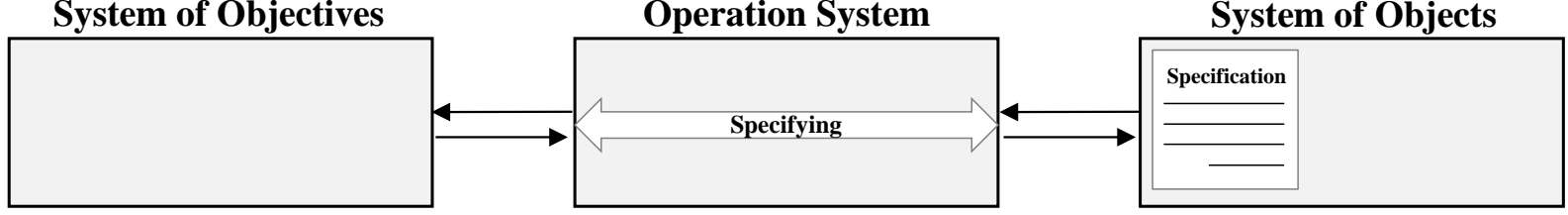

Figure 1. Correlation of specification (artifact) and specifying (activity) in the extended systemtriple approach according to (Albers, 2010)

In a recently published research paper, success factors for the specification of complex products are identified on the basis of a literature analysis and a field study and consolidated into five overarching success factors as well as transferred into an integrated reference product model (Albers et al., 2020a).

The first success factor, systems thinking, provides for the purposeful subdivision of a product into systems at different levels, taking into account the interactions associated with them, in order to do justice to the increasing networking of products through systematic decomposition. The second success factor, Stakeholder Centering, emphasizes the alignment of the development process with all stakeholders who can influence the success of the product. As a further success factor, the consideration of solution-open and solution-specific information aims at distinguishing and systematically linking specified elements of the product according to different views regarding their concretization of the technical implementation. A linkage of the element types makes a systematic and early definition and consolidation of technical requirements possible for the identification of objective conflicts, ensuring the fulfilment of requirements and avoidance of overengineering. The success factor of considering reference system elements corresponds to the basic assumption of the previously explained model of PGE - Product Generation Engineering for targeted use of existing knowledge. The last success factor is the consideration of fundamental activities of product development, which describes the dynamic development of the specification by the demand-oriented execution of activities in the development process instead of rigid process plans. By systematically linking different element types, gaps in the specification can be detected and closed by performing the corresponding activities. For example, solution-open and solution-specific elements are linked by the aforementioned activities of concretization and abstraction.

In conclusion, there is a lack of a comprehensible specification framework that differentiates between the specification as an artifact and the activity of specifying. Moreover, the full integration of identified success factors from the literature is insufficient. In particular, the link to early and continuous validation (see next section 2.2) plays a crucial role in specifying complex products.

\subsection{Continuous Validation in the Product Development Process}

Validation, as a central activity in the product development process, is the guarantor for ensuring the customer value of a product on the market (Albers, 2010; Albers et al., 2016). As an extension to verification, which answers the question "was the product developed correctly?", validation provides answers to the question "was the right product developed? (Albers et al., 2016; VDI-Fachbereich Produktentwicklung und Mechatronik, 2004). Thus, validation integrates the view of the user or customer (VDI-Fachbereich Produktentwicklung und Mechatronik, 2004). Thus, validation contributes significantly to knowledge gain during product development and supports the continuous adaptation and concretization of the system of objectives (Albers et al., 2016). A multitude of models for product development processes integrates validation activities (see Eigner et al., 2017). However, these validation activities are often strongly related to completed phases (Albers et al., 
2017a). In order to continuously integrate the results and knowledge gained from validation into the product development process and thus to expand and concretize the system of objectives, approaches for continuous validation and a parallel development of a product and the corresponding validation system are required (Albers et al., 2016; Albers et al., 2017a). In particular, dependencies between activities and (partial) results of product development and validation system development must be taken into account in order to derive findings and activities for the further development process (Albers et al., 2017a).

Overall, validation results have not yet been sufficiently taken into account in the specification in the early phase or fed back and integrated into the specification activities. In particular, it is not possible to resolve uncertain correlations and interdependencies or to identify possible reciprocal effects of different system elements in the early phase. Another factor at this point is the distributed knowledge of the product developers, which leads to a lack of transparency with regard to product models. MBSE approaches (see next section 2.3) can be used to model and represent these dependencies.

\subsection{Model-Based Systems Engineering (MBSE)}

Model-Based Systems Engineering (MBSE) comprises “[...] the formalized application of modeling to support system requirements, design, analysis, verification and validation activities, starting in the conceptual development phase and continuing through the development and later life cycle phases" (INCOSE Technical Operations, 2007, p. 15). In contrast to traditional text-based development approaches, MBSE approaches rely on the use of digital models instead of a multitude of (text) documents to capture and use relevant information, such as system specifications (Walden et al., 2015). The use of consistent models (without any logical contradictions within) promises, among other things, simplified (interdisciplinary) communication between stakeholders, potential for better control of system performance and thus increased system quality, and expanded possibilities for knowledge management and knowledge storage (Walden et al., 2015). An essential feature of MBSE is the linking of information within the models. For example, the relationship of a requirement to its implementation in the system architecture can be modeled and made traceable (see Weilkiens, 2006). This so-called "traceability" can be used, among other things, to analyze the effect of changes, for example of a requirement, on other elements, such as other requirements or elements of the system architecture. In the development and implementation of MBSE approaches, three aspects, also known as the Three Pillars of MBSE (Delligatti, 2014, pp. 4-9), must be considered in an integrated manner: the chosen modeling language, the modeling (software) tool and the selected modeling method(s). The SysML - Systems Modeling Language is widely spread in the field of MBSE (Delligatti, 2014; Object Management Group, 2017). SysML can be used to describe system requirements, system structure, system behavior and system parametrics (Delligatti, 2014). SysML offers the possibility to define new language elements as "stereotypes" via so-called profiles and thus to extend SysML (Weilkiens, 2006). Modeling methods describe how to proceed with the modeling to solve defined problems. An eample is the SYSMOD method (Weilkiens, 2006). In principle, both method and modeling language can be defined and used independently of each other and by a specific software tool. However, in order to exploit all potentials of the MBSE (especially with regard to traceability, see above), an integrated consideration and development of the three pillars of the MBSE is recommended. Some modeling methods are integrated into so-called modeling frameworks or architecture frameworks (ISO - International Organization for Standardization, 2011). These frameworks describe structuring approaches, how to structure models created with the respective methods. The goal of this structuring can be, among other things, the concretization of abstract methods in order to bring them closer to concrete application scenarios. For example, the SPES Modeling Framework structures the model two-dimensionally according to four views of the system (requirements, functional, logical and technical) and different hierarchical levels (Pohl et al., 2016). The "MagicGrid" or "MBSE Grid" also structures models two-dimensionally according to the application areas of SysML (requirements, behavior, structure, and parametric) as well as through different abstraction levels (black box, white box, solution) (Morkevicius et al., 2017; No Magic, n.d.). In the context of software engineering, the formal specification lays out functional and nonfunctional requirements (Pressman and Maxim, 2015). Therefore, a set of relevant use cases of user interactions is specified to ensure that the product functions (in this case through the implemented software) as required. Examples for formal methods of Software Engineering according to Pressman 
and Maxim, 2015 are Software Requirements Specification (SRS as in IEEE 830) or Software Design Description (SDD as in IEEE 1016).

Altogether, an insufficient applicability of "fully MBSE" approaches can be observed in practice. Furthermore, there is a high initial learning effort for modelling languages such as SysML. The existing frameworks in the literature are often designed too generically, from which the objective of this research work is concluded in the next section 3 .

\section{RESEARCH DESIGN}

A systematic and structured product specification is essential for the efficient and effective development of complex products. The previous chapter demonstrates that the explanatory model of PGE as well as further identified success factors of the specification of complex products can contribute to systematize the specification and thus make product complexity masterable. Currently, existing specification frameworks do not explicitly distinguish between the specification (in the sense of an artifact) and the activity of specifying. Furthermore, early and continuous validation insights are not fully respected and integrated into product specification activities in an early phase. Moreover, an insufficient applicability of generic MBSE approaches combined with high learning efforts for specific modelling languages poses a challenge in product development practice. Therefore, a potential of a better interdisciplinary understanding of the product specification with less error-proneness by using a central system model is observed. At the same time, however, MBSE methods are often characterized by lack of support for structuring the system model. It is therefore a promising approach and goal of this research work to integrate identified success factors in the model framework of PGE into an easily and universally applicable MBSE environment and thus make them usable as methodological support for development projects. The developed method promises to bundle the chances of existing approaches and to generate new synergies. The research work therefore further pursues the answering of the research questions (RQ) for the realization of the method (RQ1) as well as its added value (RQ2):

RQ1: How can methodological support for the specification of complex products based on current research knowledge be integrated into an MBSE environment?

RQ2: What added value does the new methodological support in MBSE generate for the specification of complex products?

To answer RQ1, the following section describes the derivation of the specification structure in MBSE as a novel methodological support in a prescriptive study. Based on the knowledge gained by applying the method in a development project, the methodological added value of the specification structure in MBSE for answering RQ2 is then critically discussed.

\section{DERIVATION OF THE SPECIFICATION STRUCTURE IN MBSE AS METHODOLOGICAL SUPPORT}

The derivation of the new methodological support in this chapter starts with the creation of the specification structure (shown schematically in Figure 2), which enables the use of identified factors in a development project in the form of a framework. Then, the transfer of the specification structure into an MBSE environment is described.

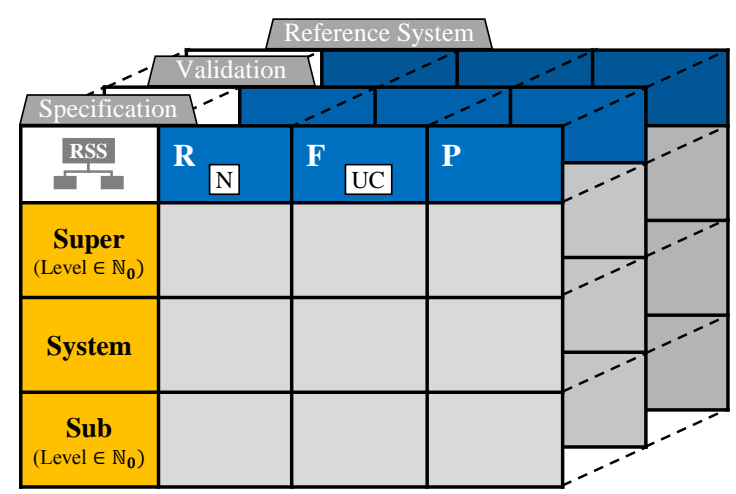

\begin{tabular}{|ll|}
\hline Legend & \\
\hline R & Requirements View \\
$\mathbf{P}$ & Functional View \\
RSS & Physical View \\
$\mathbf{N}$ & Reference System Structure (Logical View) \\
UC & Needs/Demands \\
Super & Supersystem \\
Sub & Subsystem \\
\hline
\end{tabular}

Figure 2. Derived specification structure in MBSE 
In order to take into account the first success factor, systems thinking, the specification structure allows specified elements of the product to be broken down into systems of different hierarchical levels. Similar to approaches in literature such as SPES and MagicGrid (see section 2.3), the classification along the vertical axis of the framework serves this purpose. In addition to the system level (which represents the product to be developed) the structure allows the mapping of any number of further system levels. Thereby, super systems, on hierarchically higher levels, represent the merging of systems. Subsystems denote the system decomposition and are therefore arranged on hierarchically lower levels. The vertical linking of specified elements along the hierarchical structure supports the identification of dependencies and conflicts across system levels and enables the systematic separation of product complexity. The specification structure also contains a reference system structure specifically for a concrete context, which defines the number of system levels and their respective composition from a logical point of view across projects and generations and thus enables a fast allocation of specified elements to a level.

As a further success factor, stakeholder centering is realized by integrating the validation level in the third dimension of the specification structure. This additional level supports the parallel and continuous planning and execution of validation activities in integrated consideration with the specification. The guarantee of stakeholder-centeredness within the specification is realized by linking the validation and specification level with elements of the separate artifacts needs and use cases. Regardless of the concrete manifestation in the development project, requirements represent expectations of the stakeholders (such as customers, companies, legislators) regarding the characteristics of the product. Use cases describe analogously expectations of a dynamic product usage and correspond primarily to product functions. Besides the specification level, which serves the modeling of elements of the new product to be developed, the validation level follows the same classification of views and system levels. A modeled element on the validation level extends over all system levels analogous to the elements to be validated on the specification level. Regarding the different views, the modeling on the validation level is done as follows. An element is described with respect to the defined validation objectives (requirement view), test cases (functional view) and suitable validation environments or their product models (physical view). Validation objectives are directly related to functions and requirements of the specification level. By connecting requirements and functions of the specification level to needs, use cases and customer-liable properties, a reference of the validation objectives to stakeholders is guaranteed. By linking requirements and functions to elements of the physical system architecture at the specification level, all (sub)systems involved in the fulfillment of the functions and requirements addressed by the validation target can be identified. Based on this description of the validation objectives, corresponding test cases are derived and defined to fulfill the validation objective.

The consideration of solution-open and solution-specific information is realized in the specification structure by subdividing elements into different views along the horizontal axis. Together with the logical view, in the form of the reference system structure, the subdivision according to the requirements view, functional view and physical view is based on the RFLP logic in literature as a methodology for the model-based specification of complex systems (Kleiner and Kramer, 2013). In the requirements view, objective attributes of certain product properties can be recorded. While product characteristics are of great importance due to their customer experience, they are to be characterized as open solutions due to missing description of the technical realization. Concrete technical modules and components, in the form of hardware and software, are seized however in the solution-specific physical view. This view describes thus, how the desired product is technically realized. Moderating between both views the functional view serves the description of product functions. The desired or resulting behavior of the system when fulfilling a purpose is specified. The differentiation of the three views as well as a systematic combination of the elements of different views enables a consistent specification, which puts customer-relevant requirements in direct connection with the realizing technical architecture in the form of physical elements and functions.

For the purpose of considering reference system elements, the specification structure introduces the reference system level as a further level in the third dimension. In this level all used reference system elements can be defined and, analogous to the specification level, subdivided into views and system levels. The basic principle of variation in the model of the PGE is consequently taken into account by linking elements of the reference system level with elements of the specification level. In particular, this enables the representation of intended or unintended interactions of a reference system element in 
the different system levels and views of the new product. In addition, a linkage with elements of the validation level is carried out in order to be able to represent a validation of a reference system element with regard to its use in the new product generation, if applicable.

Ultimately, the consideration of fundamental activities of product development is achieved by linking already described different elements of the specification structure via related development activities. These activities are decomposing and merging (linking elements of different system levels), concretizing and abstracting (linking elements of different views), validating (linking elements of the validation and specification levels) and varying (linking elements of the reference system and specification levels). To fill existing specification gaps in the development process, the specification structure presents the necessary activities. Thus, besides the specification as an artifact, the specification as a process of its creation is methodically guided. To support the consideration and fulfillment of previously described success factors in concrete development projects, a framework is created via the specification structure, which can serve as a structural basis for the specification of a complex product.

\section{DISCUSSION}

For evaluation and critical discussion, the presented method was applied in Live-Lab IP - Integrated Product Development. A Live-Lab represents a validation environment for design methods, in which the advantages of laboratory and field studies are used. This is done by carrying out a student development project in cooperation with a project partner from industry (Albers et al., 2018). As described in section 2.3, the integration of modeling methods into an MBSE framework offers advantages regarding the organization and structuring of the model. Accordingly, the described specification structure was implemented in the form of a modeling framework based on SysML in the software tool "No Magic Cameo Systems Modeler" (No Magic, n.d.). As described in section 4, the language elements of the SysML can be extended by stereotypes to integrate method-specific language elements. In this way, the element types described in the specification structure, which are not originally present in SysML (such as functions or validation targets), were added as stereotypes and collected in a corresponding profile. In addition to the definition of new stereotypes, possible modellable relationships between these stereotypes and the standard elements of SysML were defined in the profile. With the help of these relations it is possible to build up a continuous traceability for the newly defined stereotypes and to read them out in the software tool. By applying the method using Live-Lab IP, practical knowledge regarding applicability and benefits could be generated. The majority of the surveyed students stated that they recognized the importance of MBSE for product development. In addition, the survey showed that the modeling framework supports the understanding of the modeling approach or the corresponding method. The students perceived the modeling mainly as a means of (post-)documentation of the product development process and as a development tool for unerring development. In contrast, modeling was not understood as a support for faster progress in the development process. According to the results of the survey, an active use of model contents to support decision-making processes or communication with external parties was also rarely found. On the other hand, the survey revealed potential for the use of the modelled information and targeted, partially automated overviews for further subsequent methods, e.g. for assessing the maturity level of the system or for risk assessment of development decisions.

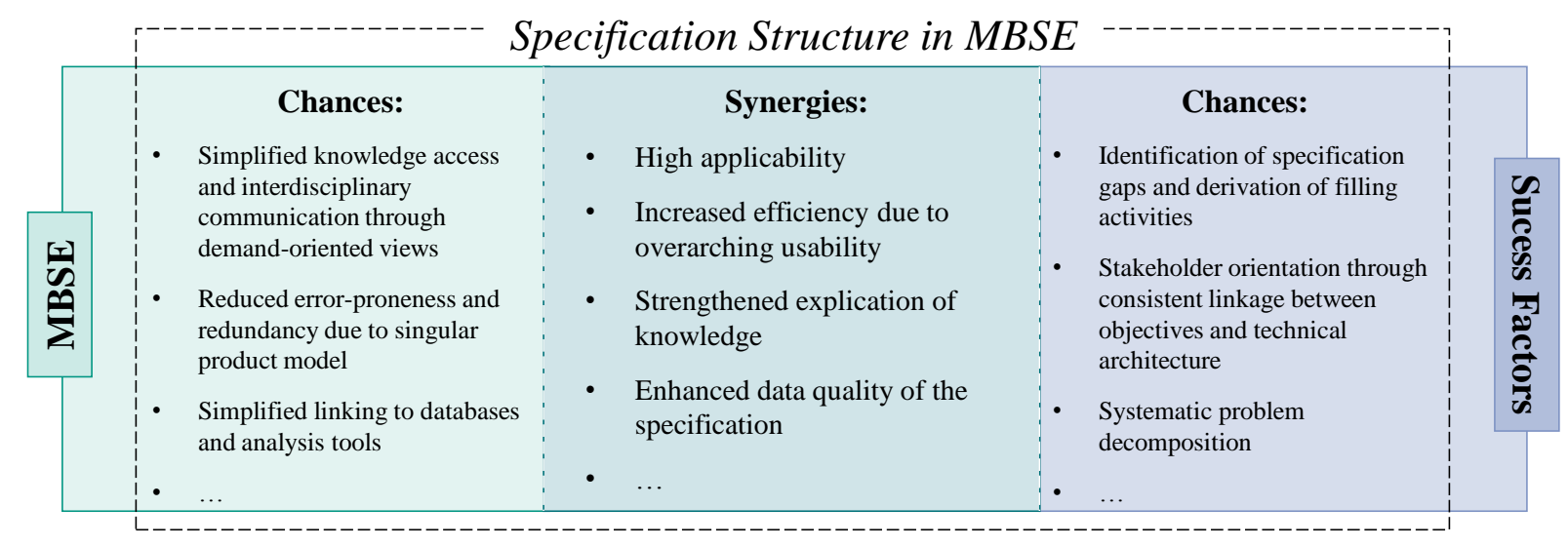

Figure 3. Opportunities of MBSE and success factors of product specification as well as novel synergies in the specification structure in MBSE 
The knowledge gained in the course of the practical application could be used to assess the added value of the presented method. It was shown that the specification structure in MBSE, by using and further developing existing approaches from the literature, in the form of success factors of the specification of complex products and the principles of MBSE, combines their opportunities, but at the same time generates novel synergies for methodological support (see Figure 3). The opportunities of existing approaches are described in the literature (see section 2.1 and 2.3). To answer RQ2, the four main synergies created will be described below. Compared to a multitude of existing MBSE methods, the presented method and the corresponding modeling framework shows a high applicability by supporting the splitting, structuring and linking of different views. Users of the specification structure can be directed to relevant views and add or extract relevant information from the model. Information that is not relevant in each case is hidden. At the same time, the use of a SysML modeling tool enables the creation of additional analysis diagrams to display views of comprehensive links of information. The possibility to extend and adapt the used modeling language SysML via profiles (see chapter 2.3) allows to adapt the views of diagrams to make relevant SysML elements directly accessible via the modeling. This adaptation of the views and the specification structure in the modeling framework make it possible that only a small amount of in-depth knowledge of SysML is required for modeling, which further increases the applicability of the method. The structure allows a systematic classification of specified elements and the derivation of activities to fill specification gaps right from the start. In addition, the method is universally applicable for different types of technical systems and allows an application and needs-based design for any development project. From this follows the synergy of efficiency increase due to comprehensive usability. A high degree of reusability across projects and product generations is ensured by the structure of specified elements, as these can be identified and found in different models if the specification structure is used consistently. This enables a seamless transfer of experience and the creation of economies of scale. A further essential synergy lies in the increased explication of implicit knowledge. The use of the central product model channels the flow of information from different departments. The specification structure, based on the success factors, guides the identification of relevant knowledge and its structured explication. Finally, a central synergy is the increased data quality of the product specification. The establishment of a uniform and structured documentation of the product from the beginning of the development process as well as the use of the standardized modeling language SysML enables the semi-automated generation of relevant diagrams for the analysis and further development of the specification. Consequently, the early detection of relevant interactions, conflicting objectives and synergies is supported. The quality and resilience of the specification can be increased without significant delay and the resulting efforts. Finally to the discussion, the missing complete evaluation of the new method in the context of an actual product development must be critically evaluated. The applications performed in the Live-Lab IP only depicted the complexity of a product as well as the underlying process of a real product development in a simplified form. However, since the new method is intended to support the mastery of product complexity, in the form of a wide variety of elements and their interactions, and process complexity, through the involvement of many different departments and other stakeholders, a practical application is essential in order to identify and verify all the added value created in the process. Due to the initial implementation, a user of the project-spanning reusability of the method could only be confirmed on the basis of the survey results among the students. The evaluation carried out within the framework of this research work thus provides practical evidence for the applicability and benefits of the new method, yet not claiming a general representation of the industry practices.

\section{CONCLUSION AND OUTLOOK}

Within the scope of the research work, the specification structure in MBSE was introduced and evaluated as a new methodological support for the specification of complex products. To derive the methodological support, success factors identified in the literature for the specification of complex products are used and transferred into a universal specification structure, which makes the success factors usable for a concrete development project. Furthermore, the specification structure was integrated into an MBSE environment in order to make the chances of using a central product model accessible for the method as well. The MBSE environment includes a corresponding method including a suitable modeling framework and its implementation in the modeling language SysML and the software tool No Magic Cameo Systems Modeler. The first practical applications of the 
specification structure in MBSE in a Live-Lab could confirm the basic applicability of the specification structure as well as the methodological added value. This added value lies in the firsttime combination of the opportunities offered by the underlying research approaches and in the creation of novel synergies that enable more efficient and effective product specification. This also represents the scientific contribution of this research work. There is potential for further research based on this work. As critically noted in the discussion, the complete evaluation of the methodological support requires an application in a real development project in order to adequately reflect actual product and process complexity. In addition, there is potential for more advanced methods that make use of the newly created structuring of specified elements. The uniform and project-spanning subdivision of elements as well as their direct linking via development activities lays the foundation for an automated design of development processes. In the course of this, a current research project of the authors is the development of a risk model, which uses a product specification structured according to the method of this research work. The risk model thereby determines standardized risk parameters from the specification and consequently derives activities for the systematic reduction of development risk.

\section{REFERENCES}

Albers, A. (2010), "Five hypotheses about engineering processes and their consequences", in Horváth, I., Mandorli, F. and Rusák, Z. (Eds.), Proceedings of the TMCE 2010 Symposium: Virtual Engineering for Competitiveness, 12.-16.04.2010, Ancona, Italien, Delft University of Technology, Delft, Netherlands, pp. 343-356.

Albers, A., Behrendt, M., Klingler, S. and Matros, K. (2016), "Verifikation und Validierung im Produktentstehungsprozess", in Lindemann, U. (Ed.), Handbuch Produktentwicklung, Carl Hanser Verlag, München, pp. 541-569.

Albers, A., Behrendt, M., Klingler, S., Reiss, N. and Bursac, N. (2017a), “Agile product engineering through continuous validation in PGE - Product Generation Engineering”, Design Science Journal, Vol. 3 No. E5, pp. 1-19.

Albers, A., Bursac, N. and Wintergerst, E. (2015), "Product Generation Development - Importance and Challenges from a Design Research Perspective", New Developments in Mechanics and Mechanical Engineering, pp. 16-21.

Albers, A., Hirschter, T., Fahl, J., Wöhrle, G., Reinemann, J. and Rapp, S. (2020a), “Generic reference product model for specifying complex products by the example of the automotive industry", in Horváth, I. and Keenaghan, G.N. (Eds.), Digital Proceedings of TMCE 2020: Designing and engineering of smart systems, 11.-15.05.2020, Dublin, Ireland, Delft University of Technology, Delft, Netherlands, pp. 353-370.

Albers, A., Klingler, S. and Ebel, B. (2013), "Modeling Systems of Objectives in Engineering Design Practice", in Lindemann, U., Venkataraman, S., Kim, Y.S., Lee, S.W., Clarkson, J. and Cascini, G. (Eds.), DS 75: Proceedings of the 19th International Conference on Engineering Design (ICED13): Design Information and Knowledge, 19.-22.08.2013, Seoul, Korea, The Design Society, Glasgow, Scotland, UK, pp. 379-388.

Albers, A., Rapp, S., Birk, C. and Bursac, N. (2017b), "Die Frühe Phase der PGE -

Produktgenerationsentwicklung”, in Binz, H., Bertsche, B., Bauer, W., Spath, D. and Roth, D. (Eds.), Stuttgarter Symposium für Produktentwicklung (SSP) 2017: Produktentwicklung im disruptiven Umfeld, 28.-29.06.2017, Stuttgart, Fraunhofer Verlag, Stuttgart, pp. 345-354.

Albers, A., Rapp, S., Fahl, J., Hirschter, T., Revfi, S., Schulz, M., Stürmlinger, T. and Spadinger, M. (2020b), "Proposing a Generalized Description of Variations in Different Types of Systems by the Model of PGE Product Generation Engineering”, in Marjanović, D., Štorga, M., Škec, S., Bojčetić, N. and Pavković, N. (Eds.), DS 102: Proceedings of the DESIGN 2020: 16th International Design Conference, 26.-19.10.2020, Dubrovnik, Croatia, Cambridge University Press, Cambridge, UK, pp. 2235-2244.

Albers, A., Rapp, S., Spadinger, M., Richter, T., Birk, C., Marthaler, F., Heimicke, J., Kurtz, V. and Wessels, H. (2019), "The Reference System in the Model of PGE: Proposing a Generalized Description of Reference Products and their Interrelations", in Wartzack, S. and Schleich, B. (Eds.), DS 94: Proceedings of the Design Society: 22nd International Conference on Engineering Design (ICED19): Responsible Design for Our Future, 05.-08.08.2019, Delft, Netherlands, Cambridge University Press, Cambridge, UK, pp. 1693-1702.

Albers, A., Walter, B., Wilmsen, M. and Bursac, N. (2018), "Live-Labs as real-world validation environments for design methods", in Marjanović, D., Štorga, M., Škec, S., Bojčetić, N. and Pavković, N. (Eds.), DS 92: Proceedings of the DESIGN 2018: 15th International Design Conference, 21.-24.05.2018, Dubrovnik, Croatia, The Design Society, Glasgow, Scotland, UK, pp. 13-24.

Alt, O. (2019), "Modellbasiertes Systems Engineering ohne Anlernaufwand mit den Fundamental Modeling Concepts”, in Schulze, S.-O., Tschirner, C., Kaffenberger, R. and Ackva, S. (Eds.), Tag des Systems Engineering (TdSE) 2019, 06.-08.11.2019, München, Gesellschaft für Systems Engineering (GfSE), Ottobrunn, pp. 205-212. 
Arslan, M., Haug, F., Heitger, N., Krämer, L. and Albers, A. (2016), “Don’t get stuck in complexity. Coping with strategic complexity in the context of Product Generation Engineering", in RADMA (Ed.), From Science to Society: Innovation and Value Creation, 03.-06.07.2016, Cambridge, UK, RADMA, Chester, UK, n. p.

D’Ambrosio, J. and Soremekun, G. (2017), "Systems engineering challenges and MBSE opportunities for automotive system design”, in IEEE (Ed.), Proceedings of IEEE International Conference on Systems, Man and Cybernetics (SMC), 05.-08.10.2017, Banff, AB, Canada, IEEE, Piscataway, NJ, USA, pp. 2075-2080.

Darlington, M.J. and Culley, S.J. (2002), "Current Research in the Engineering Design Requirement. Proceedings of the Institution of Mechanical Engineers, Part B: Journal of Engineering Manufacture", Proceedings of the Institution of Mechanical Engineers, Part B: Journal of Engineering Manufacture, Vol. 216 No. Issue 3, pp. 375-388.

Delligatti, L. (2014), SysML distilled: A Brief Guide to the Systems Modeling Language, Addison-Wesley, Upper Saddle River, NJ, USA.

Deubzer, F. and Lindemann, U. (2009), "Networked Modelling. Use and interaction of product models and methods during analysis and synthesis", in Norell Bergendahl, M., Grimheden, M., Leifer, L., Skogstad, P. and Lindemann, U. (Eds.), DS 58: Proceedings of ICED 09: 17th International Conference on Engineering Design, 24.-27.08.2009, Palo Alto, CA, USA, The Design Society, Glasgow, Scotland, UK, 371-380.

Ehrlenspiel, K. and Meerkamm, H. (2013), Integrierte Produktentwicklung: Denkabläufe, Methodeneinsatz Zusammenarbeit, 5., überarbeitete und erweiterte Auflage, Carl Hanser Verlag, München.

Eigner, M., Koch, W. and Muggeo, C. (2017), Modellbasierter Entwicklungsprozess cybertronischer Systeme: Der PLM-unterstützte Referenzentwicklungsprozess für Produkte und Produktionssysteme, Springer Vieweg, Berlin, Heidelberg.

INCOSE Technical Operations (2007), Systems Engineering Vision 2020, INCOSE-TP-2004-004-02, http://www.incose.org/.

ISO - International Organization for Standardization (2011), Systems and software engineering: Architecture description No. 42010:2011(E), ISO, Genf, Schweiz.

Kleiner, S. and Kramer, C. (2013), "Model Based Design with Systems Engineering Based on RFLP Using V6", in Abramovici, M. and Stark, R. (Eds.), Smart Product Engineering: Proceedings of the 23rd CIRP Design Conference, 11.-13.03.2013, Bochum, Springer, Berlin, Heidelberg, pp. 93-102.

Morkevicius, A., Aleksandraviciene, A., Mazeika, D., Bisikirskiene, L. and Strolia, Z. (2017), "MBSE Grid. A Simplified SysML-Based Approach for Modeling Complex Systems", in INCOSE (Ed.), Proceedings of INCOSE International Symposium 2017, 15.-20.07.2017, Adelaide, Australia, John Wiley \& Sons, Hoboken, NJ, USA, pp. 136-150.

Nellore, R. and Söderquist, K. (2000), "Strategic outsourcing through specifications”, Omega, Vol. 28 No. Issue 5, pp. 525-540.

Nellore, R., Söderquist, K. and Eriksson, K.-A. (1999), “A Specification Model for Product Development”, European Management Journal, No. Vol. 17, Nr. 1, pp. 50-63.

No Magic, I. (n.d.), “Cameo Systems Modeler”, available at: https://www.nomagic.com/products/cameosystems-modeler (accessed 7 January 2021).

Object Management Group (OMG) (2017), “OMG Systems Modeling Language. Version 1.5”, available at: https://www.omg.org/spec/SysML/1.5 (accessed 7 January 2021).

Patzak, G. (1982), Systemtechnik: Planung komplexer innovativer Systeme, Grundlagen, Methoden, Techniken, Springer-Verlag, Berlin, Heidelberg.

Pohl, K., Broy, M., Daembkes, H. and Hönninger, H. (Eds.) (2016), Advanced Model-Based Engineering of Embedded Systems: Extensions of the SPES 2020 Methodology, Springer International Publishing, Cham, Schweiz.

Pressman, R.S. and Maxim, B.R. (2015), Software Engineering: A Practitioner's Approach, 8.th ed., McGrawHill Education, New York, NY, USA.

Schuh, G. and Schwenk U (2001), Produktkomplexität managen, Hanser-Verlag, München.

Simon, H.A. (1994), Die Wissenschaften vom Künstlichen, Springer, Wien.

Smith, P. and Reinartsen, D. (1991), Developing products in half the time, Van Nordstrand Reinold, New York.

VDI-Fachbereich Produktentwicklung und Mechatronik (2004), VDI 2206: Entwicklungsmethodik für mechatronische Systeme, 03.100.40; 31.220 No. 2206:2004-06, Beuth Verlag, Berlin.

Walden, D.D., Roedler, G.J., Forsberg, K., Hamelin, R.D., Shortell, T.M. and International Council on Systems Engineering (Eds.) (2015), Systems engineering handbook: a guide for system life cycle processes and activities ; INCOSE-TP-2003-002-04, 2015, Wiley, Hoboken, NJ.

Weilkiens, T. (2006), Systems Engineering mit SysML/UML: Modellierung, Analyse, Design, dpunkt.verlag, Heidelberg. 